\title{
Design of synthetic EGR and simulation study of the effect of simplified formulations on the ignition delay of isooctane and n-heptane
}

\author{
José M. Desantes $^{\mathrm{a}}$, J. Javier López ${ }^{\mathrm{a}, *}$, Santiago Molina ${ }^{\mathrm{a}}$, Darío \\ López-Pintor ${ }^{\mathrm{a}}$ \\ ${ }^{a}$ CMT-Motores Térmicos \\ Universitat Politècnica de València \\ Camino de Vera, s/n. 46022 Valencia, SPAIN
}

\begin{abstract}
A method to create synthetic mixtures that simulate the Exhaust Gas Recirculation (EGR) of an internal combustion engine, using $\mathrm{O}_{2}, \mathrm{~N}_{2}, \mathrm{CO}_{2}, \mathrm{H}_{2} \mathrm{O}$ and Ar, has been designed. Different simplifications of this synthetic EGR have been validated in order to reproduce ignition delays. To do this, a parametric study has been carried out with CHEMKIN. The ignition delay of each simplified mixture and the ignition delay of the complete mixture have been simulated for different initial pressures, temperatures, equivalence ratios, oxygen mass fractions and for two different fuels, isooctane and nheptane. The results obtained with each simplification have been compared with the results obtained with the complete EGR, and based on this comparison the errors in ignition delay have been calculated. The behavior of the errors in ignition delay with the variation of the different parameters of the simulations has been studied. In summary, it can be seen that the relative
\end{abstract}

${ }^{*}$ Corresponding author

Tel: +34963879 232. Fax: +34963877 659. E-mail: jolosan3@mot.upv.es 
error increases with temperature and decreases with pressure, equivalence ratio and oxygen mass fraction. Finally, the limit oxygen mass fractions for the use of each simplification have been obtained. Based on these results, it can be concluded that the only gas that can be obviated to keep the error in ignition delay under $1 \%$ is Ar.

Keywords: CHEMKIN, synthetic EGR, ignition delay, NTC, PRF

\section{Introduction}

In internal combustion engines, Exhaust Gas Recirculation (EGR) is a widely used technique to reduce the formation of nitrogen oxides $\left(\mathrm{NO}_{x}\right)$ through the thermal way [1]. The working principle of EGR is to recirculate a certain amount of exhaust gases to the intake manifold, mixing them with the fresh air. The burned gases act as a diluent of the unburned mixture, thus a smaller initial oxygen mass fraction is reached and smaller combustion temperatures are obtained. In fact, the absolute temperature reached after combustion varies inversely with the exhaust gas mass fraction. Hence increasing the exhaust gas fraction reduces $\mathrm{NO}_{x}$ emissions levels [2]. This is a compulsory strategy for compression ignition (CI) engines and its use is also widespread in current spark ignition (SI) engines. Moreover, the relevance of EGR is even higher in the frame of new combustion modes, such as Homogeneous Charge Compression Ignition (HCCI), Premixed Charge Compression Ignition (PCCI) and Low Temperature Combustion (LTC). These new lowtemperature combustion strategies use massive amounts of EGR to reduce the maximum temperature reached in the cycle [3]. In this way, the soot and $\mathrm{NO}_{x}$ formation peninsulas, which can be seen in fuel/air equivalence ra- 
tio - temperature diagrams, can be avoided [4]. New combustion modes are based on the autoignition of a reactive mixture, with a higher or a lower degree of homogeneity, in an environment with low oxygen content (much less than in the atmosphere). These modes show virtually zero emissions of soot and $\mathrm{NO}_{x}$, but high emissions of unburned hydrocarbons (UHC) and carbon monoxide (CO) [5]. However, both UHC and CO are easily eliminated thanks to currently available after-treatment systems, which are efficient, effective and inexpensive.

The main challenge to implement these new combustion modes in commercial reciprocating internal combustion engines is the lack of control over the auto-ignition process and over the heat release rate. [6-8]. Improving the knowledge about the auto-ignition phenomenon of reactive mixtures in low temperature conditions is necessary to reach clean, efficient and marketable engines. As said before, these conditions are reached through the use of massive EGR. That is the reason why the auto-ignition of several fuels has to be studied under diluted environments with high EGR rates. These kind of studies are not typically carried out in single-cylinder engines but in facilities such as rapid compression machines, shock tubes or combustion vessels [9-13]. The lack of enough exhaust gas flux in these machines and their way of operation require working with synthetic EGR. So, it is necessary to define an optimal composition of such synthetic EGR to recreate the same conditions that can be found in a real engine and, by this way, to obtain results with real practical application. 


\section{Justification and objective}

In this study the validity of different synthetic EGR mixtures to determine ignition delays experimentally is intended to be solved from a point of view of simulation and modeling. Simulations have been done for two different surrogate fuels, with reactivities typical of diesel fuel and gasoline: n-heptane and isooctane, respectively. Despite the fact that more sophisticated surrogate fuels for diesel and gasoline can be found, n-heptane and isooctane were chosen because extended and fully validated chemical kinetic mechanisms are avaliable for both. Moreover, n-heptane, isooctane and their blends are primary reference fuels (PRF) employed to define the octane number reference scale and they are widely used in the literature as surrogates of diesel and gasoline under engine conditions [14-17].

Ladommatos et al. [18-21] performed a thorough study about the effect of EGR on diesel combustion, including ignition delay and exhaust emissions. They classified the effects of EGR into three different types. First, the effect of decreasing the percentage of oxygen was evaluated. It reduces the $\mathrm{NO}_{x}$ emissions at the expense of rising particulate matter and unburnt hydrocarbon emissions, and it proves to be the most relevant of all the effects. Then, the chemical and thermal effects of $\mathrm{CO}_{2}$ were studied, concluding that both have only a small effect on ignition delay and emissions. Finally, the chemical and thermal effects of $\mathrm{H}_{2} \mathrm{O}$ were studied. $\mathrm{H}_{2} \mathrm{O}$ was added up to $3 \%$ in mass, resulting in a slight increase in the ignition delay due to its higher heat capacity.

Zhao et al. [22] studied, experimentally and by simulation, five effects of EGR on ignition delay, combustion duration and heat release rate (HRR): 
EGR increases the intake charge temperature since it is composed of hot burned gases, which implies shorter ignition delays, shorter combustion durations and higher peaks of HRR. EGR has higher specific heat capacity than the fresh air and it reduces the oxygen concentration, which implies longer ignition delays, longer combustion durations and lower peaks of HRR. Chemical reactions involving the $\mathrm{CO}_{2}$ and the $\mathrm{H}_{2} \mathrm{O}$ of EGR slightly reduce the combustion duration at high concentrations of burned gases. Finally, the stratification of the burned gases from the fuel/air mixture reduces the ignition delay due to the presence of higher temperature zones at the boundary between the hot burned gases and the unburnt charge. Dec [23] also performed a computational study of the EGR effects on the heat release rate using isooctane, obtaining that the higher the EGR rate, the smoother the HRR curve.

Chen et al. [24] studied the effect of EGR by simulation, using internal combustion engine models from CHEMKIN fuelled with methane. They divided the effects of the EGR into three: the thermal factor (referred to the high temperature of the hot EGR), the dilution factor and the chemical factor. Their results agree with the results of Zhao et al. Of course, the overall effect of the hot EGR depends on the temperature of the gases and the degree of dilution together with the chemical effects. The composition of the EGR used by Chen et al. was fixed and equal to $79 \% \mathrm{~N}_{2}, 15.5 \% \mathrm{H}_{2} \mathrm{O}, 5 \%$ $\mathrm{CO}_{2}$ and $0.5 \% \mathrm{O}_{2}$, which provides quite realistic results. However, different fuels and different EGR compositions should be studied.

Sjöberg et al. [25] performed a similar study where autoignition results of PRF blends with different diluents were compared. The experiments were 
carried out using a single-cylinder engine and by simulation with CHEMKIN. They studied three EGR effects: thermodynamic cooling effect due to the increased specific-heat capacity of the mixture and $\mathrm{O}_{2}$ reduction effect, which increase ignition delays; and chemical effects. Sjöberg et al. considered two chemical effects of EGR: the $\mathrm{H}_{2} \mathrm{O}$ chemical effect that enhances autoignition, and the effect of trace species, such as unburned or partially oxidized hydrocarbons, that enhance or suppress autoignition depending on the type of fuel. The study of Sjöberg et al. was completed afterwards with ethanol [26] obtaining similar results.

A complete CFD study about the thermodynamic effects of EGR was performed by Babajimopoulos et al. [27] using KIVA-3V. Both the influence of the EGR temperature on the heat losses and the degree of EGR stratification (temperature and composition heterogeneities) were analyzed in a natural gas engine for different variable valve actuation strategies. These EGR thermodynamic effects in natural gas engines were also studied by Fathi et al. [28] concluding that the thermal losses decrease if the percentage of cold EGR increases because lower temperatures are reached.

Cong and Dagaut [29] studied the effect of $\mathrm{CO}_{2}$ on methane oxidation in a jet-stirred reactor at low pressures $(0.1$ to $1 \mathrm{MPa})$ and medium-to-high temperatures (900 to $1400 \mathrm{~K}$ ). These authors found a slight increase of the ignition delay working with $20 \%$ of $\mathrm{CO}_{2}$. Herzler and Naumann [30] employed a shock tube to study the effects of $\mathrm{NO}_{x}, \mathrm{H}_{2} \mathrm{O}$ and $\mathrm{CO}_{2}$ on the ignition delay of natural gas at 1.6 MPa and high temperatures (1000 to $1700 \mathrm{~K}$ ). They found that even small amounts of $\mathrm{NO}_{x}$ lead to significantly shorter ignition delays. No significant influence on the ignition delay with $\mathrm{H}_{2} \mathrm{O}$ volume frac- 
tions of 30 and $50 \%$ was found because the promoting chemical effect of $\mathrm{H}_{2} \mathrm{O}$ on ignition is balanced by its higher heat capacity. Moreover, an increase of the ignition delay is found with $30 \%$ of $\mathrm{CO}_{2}$ due to its higher heat capacity (at low temperatures) and due to relevant chemical effects (at high temperatures). Finally, Di et al. [31] studied the effects of gas composition on low temperature ignition of isooctane and n-heptane computationally and with a rapid compression machine, working with $\mathrm{N}_{2}$, Ar and a blend of $\mathrm{Ar}$ and $\mathrm{CO}_{2}$. Their results showed that thermal effects are very pronounced and are dominant at low temperature conditions, whereas at temperatures higher than $850 \mathrm{~K}$, the chemical effects of $\mathrm{CO}_{2}$ became more important than the thermal ones.

From this literature survey, it can be seen that there are a lot of studies about the effects of the EGR components on the different parameters of the combustion process. However, it is necessary to analyze these effects with realistic proportions of the species in order to define the relevance of each of them under engine conditions. The present work is justified because of the great amount of studies where the auto-ignition of different fuels at low temperatures is analyzed. In them, the ignition delay is obtained in environments poor in oxygen, and the dilution is done by adding $\mathrm{N}_{2}, \operatorname{Ar}$ or $\mathrm{CO}_{2}$ indistinctly [32-34]. This way to control the percent of oxygen is very useful to obtain ignition delays at different temperatures and pressures, and with different combustion temperatures. However, a more proper composition of the dilution gases is necessary if a more realistic study is intended to be performed. In order to obtain ignition delays under various engine conditions, the question is how to dilute the air in the simplest way, but without altering 
the reality. This study intends to give an answer to this question. Ignition delays for isooctane and n-heptane are obtained under different conditions of pressure, temperature, fuel/air equivalence ratio and oxygen mass fraction, and also for different EGR compositions. The calculations are performed with the wide-spread software for chemical kinetics simulation, CHEMKIN. This software, which is developed by Reaction Design (ANSYS), is consolidated in the world of engineering investigations and the chemical kinetics mechanisms of several hydrocarbons are perfectly defined to be used with it. This study is a work of simulation and modeling.

\section{Methodology}

\subsection{Introduction}

The methodology employed in this study is presented in this section. First, the synthetic EGR is defined, as well as the equations employed to calculate its composition. Then, the calculation software, the chemical kinetic mechanisms used and the criteria taken to define the ignition delay are described. Finally, the simplifications of the complete synthetic EGR considered are shown and justified, and the parametric study performed is described.

\subsection{Definition of the synthetic EGR}

The influence of minor components of the EGR on the ignition delay has been checked in previous studies [30, 35-37]. These minor components (UHC, CO, formaldehyde and NO) have been obviated in this study due to the great difficulty implied by adding them to a synthetic EGR mixture. 
Although NO, for instance, has a significant effect on the ignition delay, trying to use it in a synthetic EGR mixture involves complex issues. On one hand, the difficulty lies in the impossibility to know the amount to be added for each particular case, as the proportion of this component cannot be easily determined. On the other hand, if that amount is known, this species is found at a rate of a few parts per million, so that obtaining a synthetic mixture with such a precise amount would be extremely complex. Thus, the synthetic mixture which is considered as real EGR in the present study consists of the products of a complete combustion of a mixture of fuel and dry air with such a proportion of each component so as to achieve the final oxygen mass fraction desired by the user. If the minor compounds of air are neglected (those with volume fractions lower than 0.04\%), dry air may be assumed as formed by $20.954 \%$ oxygen, $78.112 \%$ nitrogen and $0.934 \%$ argon (all figures given in molar fractions). For any hydrocarbon with the form $C_{n} H_{m} O_{p}$ the complete combustion reaction with dry air can be written as:

$$
\begin{array}{r}
C_{n} H_{m} O_{p}+\frac{a_{s t}}{\phi} \cdot\left(O_{2}+\frac{0.78112}{0.20954} \cdot N_{2}+\frac{0.00934}{0.20954} \cdot A r\right) \rightarrow n C O_{2}+ \\
+\frac{m}{2} H_{2} O+\left(\frac{a_{s t}}{\phi}-n-\frac{m}{4}+\frac{p}{2}\right) O_{2}+\frac{0.78112}{0.20954} \cdot \frac{a_{s t}}{\phi} N_{2}+\frac{0.00934}{0.20954} \cdot \frac{a_{s t}}{\phi} A r
\end{array}
$$

where $a_{s t}=n+m / 4-p / 2$ and $\phi$ represents the equivalence ratio. Obviously, the factor $a_{s t} / \phi-n-m / 4+p / 2$ is higher than or equal to zero if the equivalence ratio is less than or equal to 1 , which means that this combustion reaction is only valid for lean mixtures. If it is intended to work with a given mass fraction of oxygen $\mathrm{Y}_{\mathrm{O}_{2}}$, the composition of synthetic EGR providing it 
must satisfy the following equation:

$$
Y_{O_{2}}=\frac{M_{O_{2}}}{M_{\text {products }}}=X_{O_{2}} \frac{M W_{O_{2}}}{M W_{\text {products }}}
$$

where $\mathrm{M}_{i}$ and $M W_{i}$ are the mass and the molecular weight of the species $i$, respectively, and $\mathrm{X}_{\mathrm{O}_{2}}$ can be calculated as:

$$
X_{O_{2}}=\frac{\frac{a_{s t}}{\phi}-n-\frac{m}{4}+\frac{p}{2}}{4.77236 \frac{a_{s t}}{\phi}+\frac{m}{4}+\frac{p}{2}}
$$

where all the terms are known except the necessary equivalence ratio, $\phi$, which is defined as the auxiliary equivalence ratio. $\phi$ is only used to determine the composition of the synthetic EGR and it is by no means related to the fuel/air equivalence ratio, $F r$, that later on will have the fuel + EGR mixture, as described further below. Once this auxiliary equivalence ratio is known, the determination of the composition of the synthetic EGR which provides a mixture with the desired oxygen content is quite obvious.

This method for designing synthetic EGR is based on obtaining a mixture that satisfies an objective in oxygen mass fraction. When studying the autoignition at low temperature conditions (with deficit of $\mathrm{O}_{2}$ ) it makes much more sense to talk about oxygen mass fractions rather than EGR rates. The same EGR rate, depending on the load (i.e. on the working equivalence ratio) leads to different amounts of oxygen in the intake and, therefore, to different combustion temperatures. However, the conditions of the study are perfectly determined if the mass fraction of $\mathrm{O}_{2}$ and the working equivalence ratio are used.

In connection with the previous paragraph, since the fuel is not mixed with pure air, but with a blend of air and exhaust gases with a given oxygen 
mass fraction, it is necessary to define how to compute the fuel-air ratio. For this purpose a fuel-oxygen ratio defined as $F=M_{f} / M_{O_{2}}$ was used. This concept is very useful to study the autoignition process in environments with deficit of oxygen, since it maintains the meaning of the equivalence ratio and, at the same time, it defines, uniquely, the working amount of fuel. The equivalence ratio is defined in this work as $F r=F / F_{s t}$, where st refers to stoichiometric conditions. Thus, for a certain required equivalence ratio, $\mathrm{Fr}$, the amount of fuel is given by:

$$
X_{f}=\frac{X_{O_{2}} \cdot F_{r}}{a_{s t}+X_{O_{2}} \cdot F_{r}}
$$

where $X_{\mathrm{O}_{2}}$ is the oxygen molar fraction in the synthetic EGR.

The methodology used to obtain the composition of the fuel-EGR mixture can be summarized as follows: an auxiliary equivalence ratio, $\phi$, is calculated for a desired oxygen mass fraction by using Eq. $(2,3)$. The EGR composition is defined with this auxiliary equivalence ratio and $\mathrm{Eq}(1)$. The molar fraction of fuel, $X_{f}$, is calculated for a desired working equivalence ratio, $F r$, by using Eq (4). Finally, the composition of the fuel-EGR mixture is calculated by $X_{i \text { mixture }}=X_{i}\left(1-X_{f}\right)$.

Finally, it is worthy to note that this methodology allows working with very low amounts of oxygen even if very lean mixtures are being studied, which can be not realistic. To check the realism, the EGR rate corresponding to a certain oxygen molar fraction, $X_{\mathrm{O}_{2}}$, and to a working equivalence ratio, Fr, can be estimated with sufficient accuracy by using Eq. (5), which should give values below $100 \%$. 


$$
\% E G R=\frac{X_{O_{2}}{ }_{a t m}-X_{O_{2}}}{F_{r}} \cdot 100
$$

\subsection{CHEMKIN and chemical kinetic mechanisms}

As already mentioned in the previous section, CHEMKIN is the software used to obtain the different ignition delays. The version used is CHEMKINPRO 2009. Curran's kinetic mechanism is used both for isooctane and nheptane $[38,39]$. This mechanism consists of 1034 species and 4238 reactions, and includes the chemical kinetics of the two hydrocarbons used in this investigation. Its validity has been checked in several articles [14, 15, 40] by comparison with experimental results. Besides, a simplified chemical kinetic mechanism has been used to facilitate the analysis of the reactions where the species of the synthetic EGR are involved in. This latter mechanism used was developed by Patel [41] and it consists of 29 species and 52 reactions. The validity of this mechanism was proven in the given reference by comparison with Curran's mechanism. Whereas Patel's mechanism is used only to determine the most relevant reactions for this study, Curran's mechanism is used to perform all the calculations.

The model used is a homogeneous closed reactor (perfectly stirred reactor, PSR), which works with constant pressure, and uses the energy equation to solve the temperature temporal evolution. This model is the most appropriate to obtain ignition delays under certain pressure and temperature conditions [42]. Besides, working with constant pressure corrects, somehow, the over-prediction of the pressure which is typical of this kind of reactor [43]. 
In this work the auto-ignition of the mixture is considered to be produced when the temperature in the reactor increases $400 \mathrm{~K}$ over the initial temperature ( $\Delta T$ criterion). The time corresponding to this instant will be considered as the ignition delay, $\tau$. This criterion is widely recognized by the scientific community $[43,44]$. Although the criterion chosen could seem inappropriate, since the ignition delay seems to be referenced to different phases of the combustion for different simulations, its consistency with other criteria has been checked for both fuels and various equivalence ratios $(F r \in\{0.5,1$, $2\})$, pressures $(P \in\{5,10,15\} M P a)$, temperatures $(T \in\{700,800,900$, $1000\} K)$ and oxygen mass fractions $\left(Y_{\mathrm{O}_{2}} \in\{0.21,0.15\}\right)$. For each case, the ignition delay is obtained from the $\Delta T$ criterion and from the criterion of using the time at which the maximum temperature gradient occurs $(\nabla T$ criterion).

In Fig. 1, ignition delays are represented, those obtained from the $\Delta T$ criterion in the ordinates axis and those obtained from the $\nabla T$ criterion in the abscissas axis. The graph on the left shows the results obtained for isooctane whereas the graph on the right shows the results obtained for n-heptane. The line $\mathrm{y}=\mathrm{x}$, which represents a perfect match between both criteria, is plotted in all graphics. Both figures show a good agreement between both criteria. In fact, the coefficients of determination, $\mathrm{R}^{2}$, has been calculated for each fuel and each method and their values can be seen in the corresponding figure.

Ignition delays are always slightly underpredicted by using the $\Delta T$ criterion instead of using the $\nabla T$ criterion. However, this underprediction is lower than $5 \%$ in case of using n-heptane and lower than $2.5 \%$ in case of using isooctane. Moreover, the difference between both criteria has always 

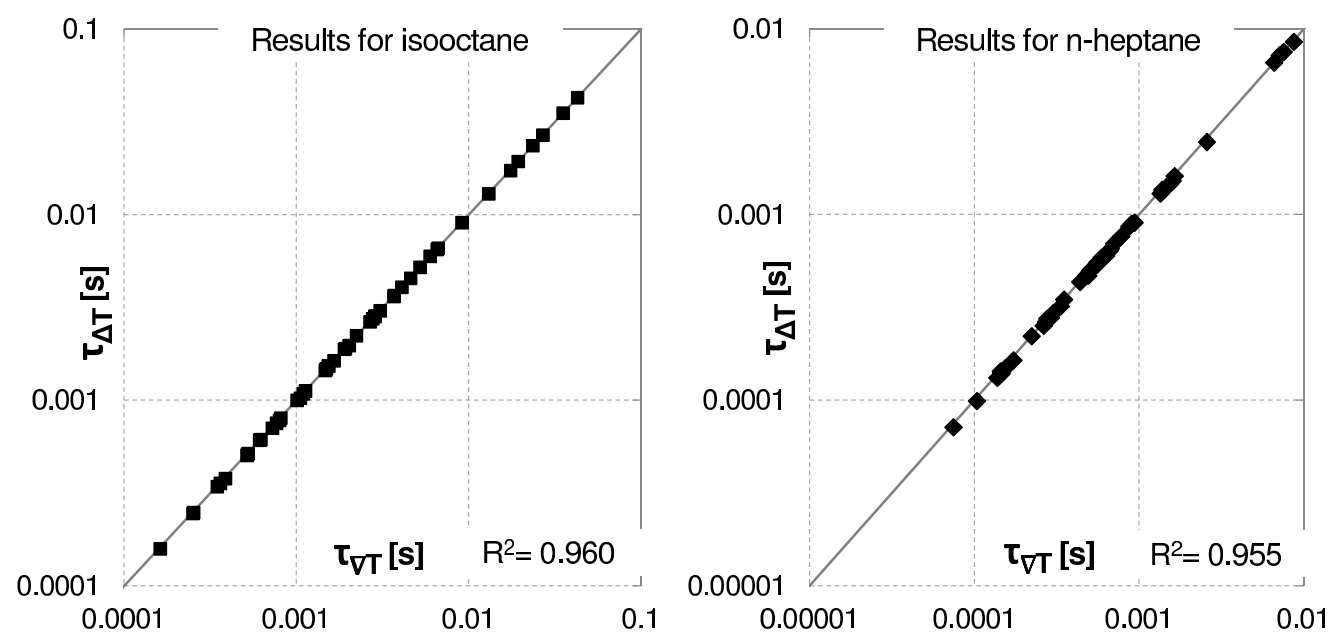

Figure 1: Ignition delays obtained from the $\Delta T$ criterion versus ignition delays obtained from the $\nabla T$ criterion. All cases in which autoignition occurs are represented. Left.Isooctane. Right.- n-Heptane.

the same trend, which guarantees the correct interpretation of the results. Finally, the $\Delta T$ criterion is chosen in this study because of its higher stability compared to other criteria, which can lead to more than one result for the ignition delay in certain simulations.

The maximum waiting time for the auto-ignition of the mixture has been set to $30 \mathrm{~ms}$. This value has been chosen because higher ignition delays would be equivalent to combustions after top dead center (TDC) in an engine operating at $1000 \mathrm{rpm}$ or higher. Therefore, mixtures with ignition delays longer than $30 \mathrm{~ms}$ have no interest in the context of automotive engines.

3.4. Simplifications of the complete synthetic EGR and parametric study performed

The different types of synthetic EGR evaluated were the following: 
- Mixture formed by $\mathrm{O}_{2}, \mathrm{~N}_{2}, \mathrm{CO}_{2}, \mathrm{H}_{2} \mathrm{O}$ and Ar: it is called real EGR and it is formed by all the species except the minor compounds (NO, $\mathrm{CO}$, UHC and formaldehyde), which have complex reproducibility, as already mentioned before.

- Mixture formed by $\mathrm{O}_{2}, \mathrm{~N}_{2}, \mathrm{CO}_{2}$ and $\mathrm{H}_{2} \mathrm{O}$ : it was decided to obviate the argon because it is, typically, the minor compound of the real EGR mixture.

- Mixture formed by $\mathrm{O}_{2}, \mathrm{~N}_{2}$ and $\mathrm{CO}_{2}$ : it was is decided to obviate the water due to the difficulty in adding it to the mixture. Water is the only species in use that is liquid at ambient temperature, and since the metering in vapor state is complex, it is preferable to work with it in liquid phase. This implies that a system is needed for $\mathrm{H}_{2} \mathrm{O}$ injection and also for its evaporation. In case of working with a synthetic EGR without water, it would be possible to prepare the mixture more easily with a filling based on partial pressures, since all the components are gases.

- Mixture formed by $\mathrm{O}_{2}, \mathrm{~N}_{2}$ and $\mathrm{H}_{2} \mathrm{O}$ : the carbon dioxide was obviated due to the difficulty in adding it to the mixture when the oxygen content is similar to the atmospheric one. The higher the mass fraction of oxygen, the smaller the amount of $\mathrm{CO}_{2}$ to be added and, therefore, the lower the partial pressure of $\mathrm{CO}_{2}$ in the mixture. Thus, if a filling based on partial pressures is done, it is necessary to have an initial vacuum pressure lower than the lowest partial pressure of the mixture. Moreover, a high enough resolution in the pressure sensor used to con- 
trol the filling is needed. For all these reasons, using $\mathrm{CO}_{2}$ under certain conditions can be really difficult.

- Mixture formed by $\mathrm{O}_{2}$ and $\mathrm{N}_{2}$ : it is the simplest form of diluting the amount of oxygen, by using nitrogen, the major component both in the ambient air and in the exhaust gases of an engine.

For each of the four types of mixtures in which some of the species are obviated, the value of its molar fraction was added into the molar fraction of nitrogen. Thus, the oxygen ratio desired by the user is conserved.

The validity of each of these mixtures was checked by comparing the ignition delays obtained with CHEMKIN with the ignition delays obtained for the real EGR mixture. This comparison was carried out at different equivalence ratios, oxygen mass fractions, temperatures and pressures, and it was done with both isooctane and n-heptane. The performed parametric study was as follows:

- Temperature: from $600 \mathrm{~K}$ to $1100 \mathrm{~K}$, at steps of $50 \mathrm{~K}$.

- Pressure: from $5 \mathrm{MPa}$ to $15 \mathrm{MPa}$, at steps of $2.5 \mathrm{MPa}$.

- Fuel/air equivalence ratio: from 0.25 to 2.5 , at steps of 0.25 .

- Oxygen mass fraction: 0.2285 (ambient air, without EGR) and from 0.21 to 0.09 , at steps of 0.02 .

leading to a final number of 4400 cases to be compared, for each fuel and each type of synthetic EGR. 
Most of the operating range of internal combustion engines is covered with this parametric study. Although equivalence ratios of 2.5 can seem uninteresting for practical applications, it must be taken into account that autoignition occurs under rich local equivalence ratios in direct-injection diesel engines [45]. This concept is known as most reactive mixture fraction and it arises due to the balance of reactivities between the fuel-air ratio distribution and the temperature distribution. Depending on the working conditions, local equivalence ratios on the order of 2 can be the most reactive ones.

\section{Results and discussion}

In this section, the range of application of each of the previous simplifications is evaluated, which is the main objective of this study. First, the validity of the simulations will be checked. For this reason the trends and orders of magnitude of the ignition delays will be analyzed, since they must be congruent with the auto-ignition theory. Second, the relative error in ignition delay will be defined. This parameter is the one that will be used to decide about the validity of each synthetic mixture. The variations of this error with the different parameters of the simulation (initial temperature, pressure, equivalence ratio and oxygen mass fraction) will be analyzed. Finally, a maximum admissible error will be taken and, based on this, the ranges of validity of each simplified mixture will be established.

As expected $[33,46]$, the following trends in the ignition delay were observed (Fig. 2):

- Ignition delay decreases when the initial temperature is increased. For n-heptane, this is the case except in the negative temperature coefficient 
zone, NTC. In the parametric study performed, and if other parameters such as pressure, equivalence ratio and oxygen fraction are obviated, the ranges of the NTC zone are the following:

- Isooctane: between $800 K$ and $900 K$.

- n-Heptane: between $850 K$ and $1050 K$.
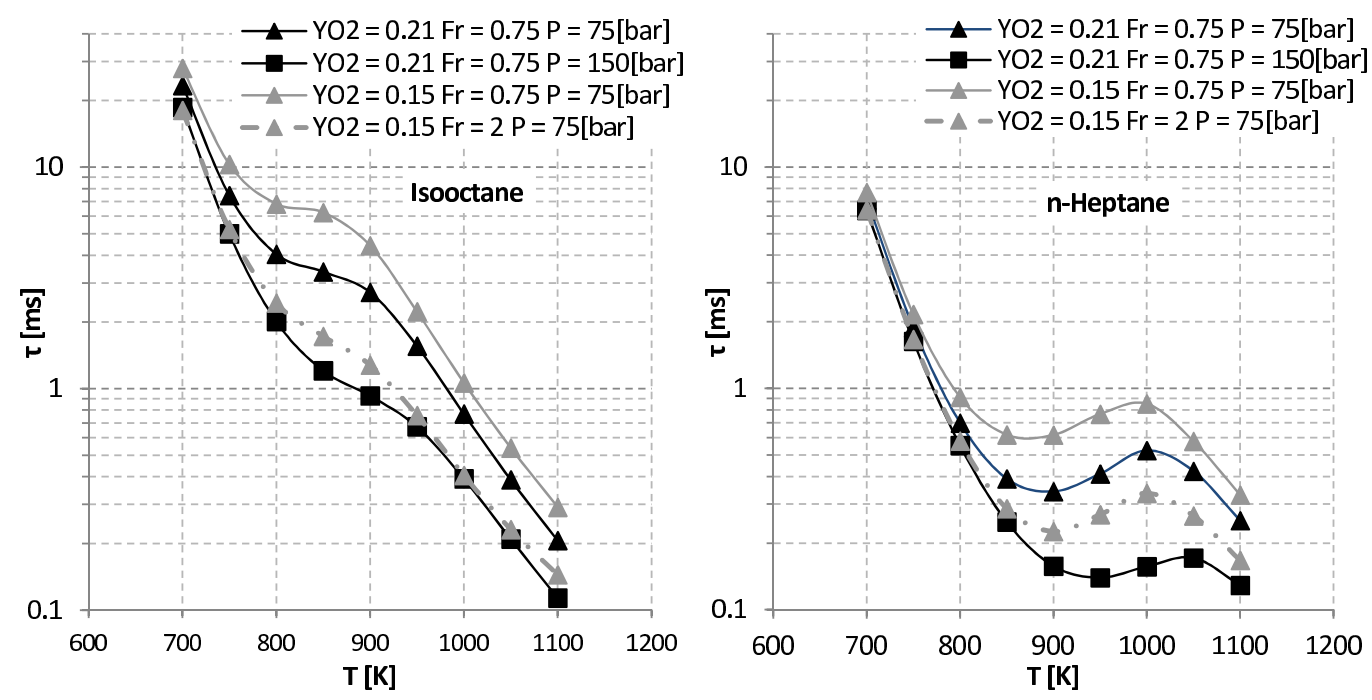

Figure 2: Ignition delay versus temperature for different conditions. Left.- Isooctane. Right.- n-Heptane.

Moreover, it can be seen that the NTC zone is moved to higher temperatures and it becomes less pronounced if the pressure is increased. In the same way, it can be seen that the NTC zone is moved to lower temperatures and it becomes more pronounced if the oxygen proportion is reduced. Besides, the NTC zone is moved to higher temperatures and it becomes less pronounced if the equivalence ratio is increased. Finally 
and as expected, the NTC zone of the n-heptane is more pronounced than the NTC zone of the isooctane.

- Ignition delay decreases when the pressure is increased for all the simulated range and for both fuels.

- Ignition delay increases when the mass fraction of oxygen of the mixture is decreased. Lower amount of oxidizer implies minor reactivity.

- Ignition delay decreases when the equivalence ratio is increased in the explored range.

These trends can be seen for the four types of synthetic EGR.

An error in ignition delay has been obtained for each case. For each synthetic mixture $i$ at some given conditions of pressure, temperature, equivalence ratio and mass fraction of oxygen, this error is calculated as follows:

$$
\xi_{i}=\frac{\tau_{i}-\tau_{\text {realEGR }}}{\tau_{\text {realEGR }}} \cdot 100
$$

where realEGR corresponds to the mixture formed by $\mathrm{O}_{2}, \mathrm{~N}_{2}, \mathrm{CO}_{2}, \mathrm{H}_{2} \mathrm{O}$ and Ar.

The behavior of this error as a function of various parameters will be analyzed now. First, how the error varies with the initial temperature is studied, as well as the causes of this variation. Then, how it varies with the equivalence ratio and with the oxygen mass fraction of the synthetic mixture will be analyzed.

As expected, the smallest error is obtained in the EGR formed by $\mathrm{O}_{2}+$ $\mathrm{N}_{2}+\mathrm{CO}_{2}+\mathrm{H}_{2} \mathrm{O}$, and it increases as additional species are obviated. As a consequence the highest errors are found in the mixture formed by $\mathrm{O}_{2}+\mathrm{N}_{2}$. 
In fact, argon can be obviated if the oxygen mass fractions is less than or equal to 0.09 without exceeding $1 \%$ error in ignition delay.

\subsection{Effect of the initial temperature on the ignition delay error}

The effect of the initial temperature on the ignition delay error is shown in Fig. 3, 4 and 5. The error in ignition delay increases when the initial temperature is increased, up to a maximum. The behavior of the error with the temperature variation is justified by two effects, a thermodynamic effect and a chemical effect. As will be explained below, both effects cause opposite effects on the ignition delay error. Thus, a maximum in the ignition delay error (a change of trend in the evolution of the error) implies a change in the relevance of the effects. The location of this maximum is affected by the following trends, all of them shown in Fig. 3:

- The higher the equivalence ratio, the higher the initial temperature where this maximum error is located. In fact, in some cases this maximum cannot be seen.

- The maximum error in ignition delay is also displaced to higher initial temperatures when either the pressure (Fig. 5) or the oxygen content are increased.

- The initial temperature at which the maximum error in ignition delay occurs is, for the same conditions, higher for n-heptane than for isooctane.

These trends can be seen for both fuels and for all types of EGR, as it is shown in Fig. 4 for n-heptane and Fig. 5 for isooctane. 

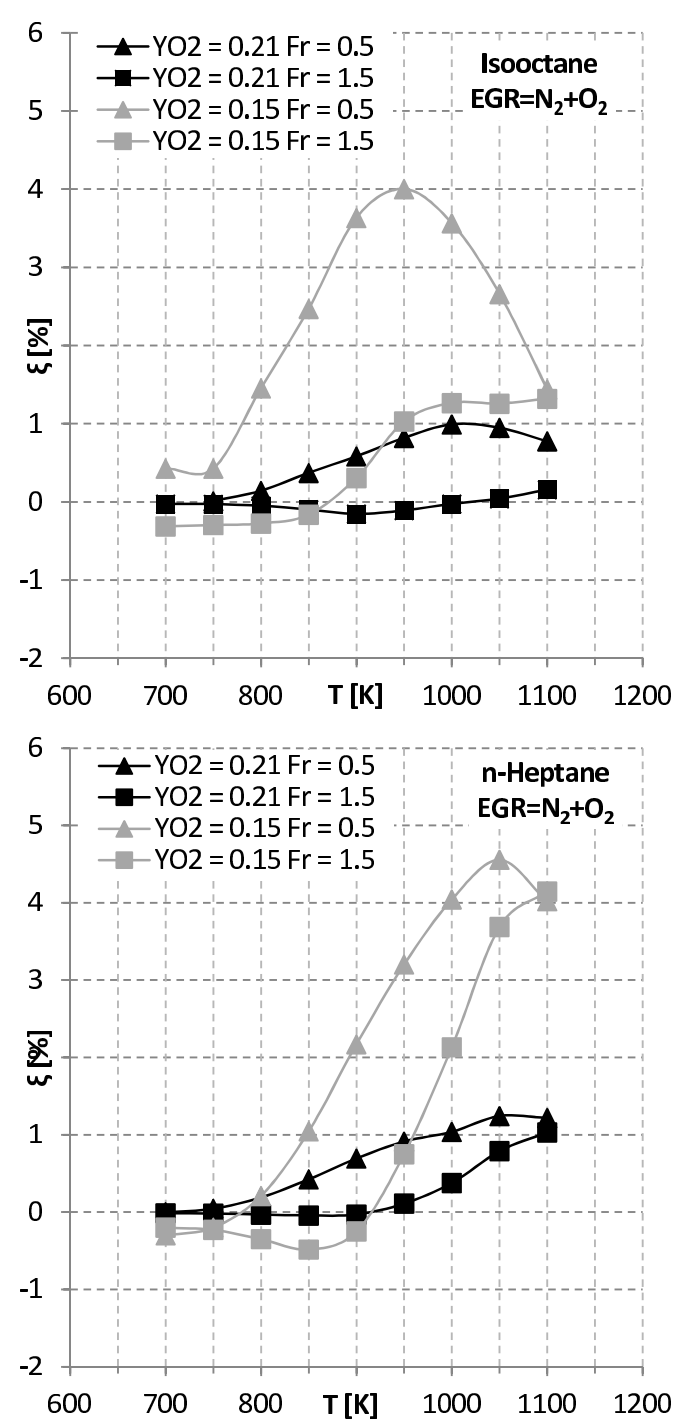

Figure 3: Ignition delay error versus temperature at $\mathrm{P}=10 \mathrm{MPa}$, different equivalence ratios and different oxygen mass fractions, which were obtained with an EGR formed by $\mathrm{O}_{2}$ and $\mathrm{N}_{2}$. Top.- Isooctane. Bottom.- n-Heptane. 


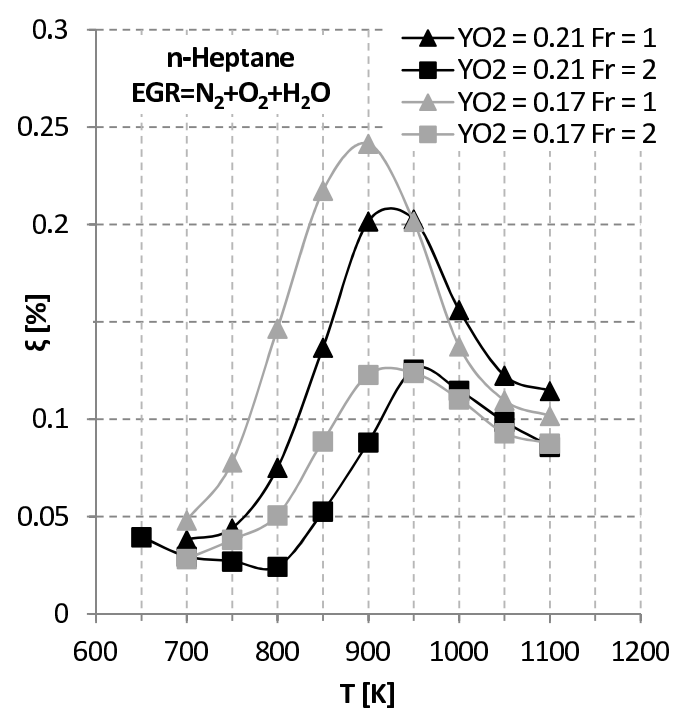

Figure 4: Ignition delay error versus temperature for n-heptane at $\mathrm{P}=10 \mathrm{MPa}$, different equivalence ratios and different oxygen mass fractions, which were obtained with an EGR formed by $\mathrm{O}_{2}+\mathrm{N}_{2}+\mathrm{H}_{2} \mathrm{O}$. 


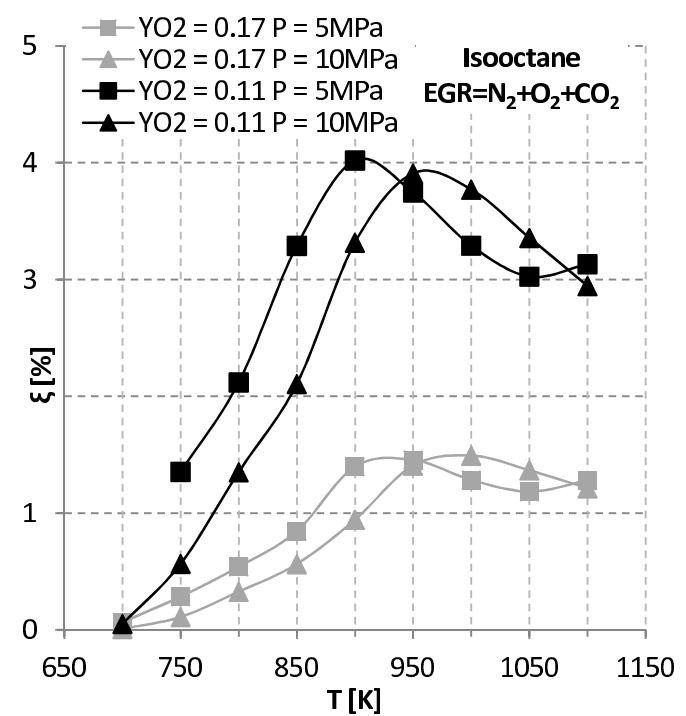

Figure 5: Ignition delay error versus temperature for isooctane at $\mathrm{Fr}=1$, different oxygen mass fractions and different pressures, which were obtained with an EGR formed by $\mathrm{O}_{2}$ $+\mathrm{N}_{2}+\mathrm{CO}_{2}$. 


\subsubsection{Thermodynamic effect}

The thermodynamic effect arises because the heat capacity of the mixture is different for each synthetic EGR. A different composition implies different heat capacity, which implies a different evolution of the temperature. Since a lower heat capacity means less heat sink effect, the mixture with a lower heat capacity also undergoes, obviating chemical aspects, a faster temperature increase. Taking into account the different heat capacities of the different species, Thermodynamics predicts lower ignition delays in those mixtures where $\mathrm{H}_{2} \mathrm{O}$ and $\mathrm{CO}_{2}$ are obviated (since both species have higher heat capacity than $\mathrm{N}_{2}$ ) and longer ignition delays in the mixture where $\mathrm{Ar}$ is obviated (since Ar has lower heat capacity than $\mathrm{N}_{2}$ ).

Moreover, the higher the initial temperature, the higher the differences in the heat capacities. This causes higher differences in the temperature evolutions and, therefore, a higher ignition delay error. Besides, for a fixed temperature error, the higher the initial temperature, the higher the differences in the specific reaction rates (due to the exponential dependency of the specific reaction rate with temperature). Finally, the errors in specific reaction rate can be directly extrapolated to ignition delay errors. These two behaviours imply that the absolute value of the ignition delay error increases with the initial temperature.

Besides, the ignition delay error caused by thermodynamic effects is also affected by the duration of the combustion. If the exothermic stage (and, consequently, the temperature increase) occurs in a shorter time interval, the differences in heat capacities become less relevant for the calculation of the ignition delay. 


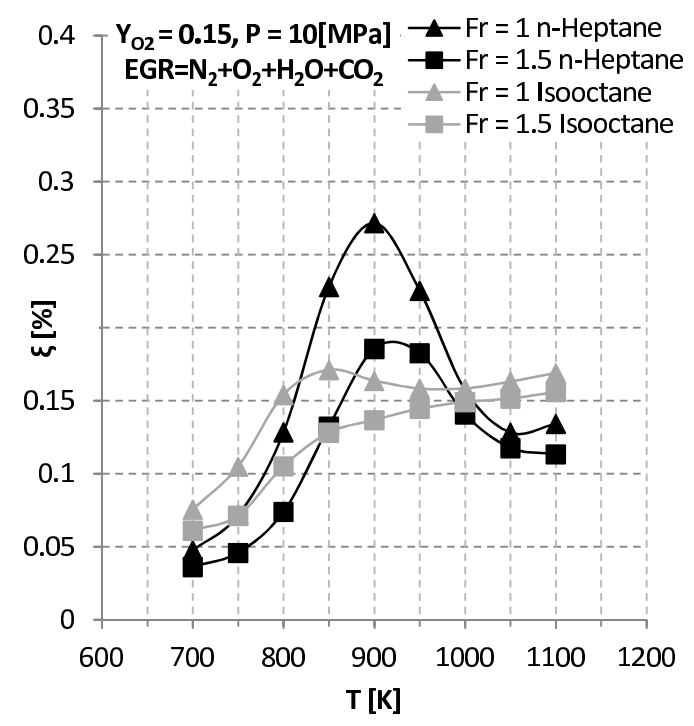

Figure 6: Ignition delay error versus temperature at $\mathrm{P}=10 \mathrm{MPa}$ and $\mathrm{Y}_{\mathrm{O}_{2}}=0.15$, different fuels and equivalence ratios, which were obtained with an EGR formed by $\mathrm{O}_{2}+\mathrm{N}_{2}+$ $\mathrm{CO}_{2}+\mathrm{H}_{2} \mathrm{O}$.

The consequences of all effects can be seen in Fig. 6, where only Ar is obviated and, therefore, only thermodynamic effects are present.

The longer the exothermic stage (two-stage ignition or NTC regime) the higher the ignition delay error. In fact, the error increases fastly in the range of temperatures where the two-stage ignition of n-heptane occurs, and when the two-stage ignition disappears (above a certain temperature), the error decreases because the duration of the combustion is highly reduced. Finally, it becomes increasing again as expected. This behavior is much less pronounced in case of using isooctane, since this fuel does not present a two-stage ignition.

Regarding the definition of the ignition delay error (Eq. (6)), the impact 
of the thermodynamic effect on it is very clear:

- If Ar is obviated the error becomes more positive as the initial temperature is increased, up to a maximum. Then, the error decreases due to the end of the two-stage ignition or the end of the NTC regime. Finally, it increases again as expected.

- If $\mathrm{H}_{2} \mathrm{O}$ or $\mathrm{CO}_{2}$ are obviated the error becomes more negative as the initial temperature is increased, up to a minimum. Then, the error becomes less negative due to the end of the two-stage ignition or the end of the NTC regime. Finally, it becomes more negative again as expected.

Despite the fact that this behavior is observed in all the range of initial temperatures when only $\mathrm{Ar}$ is obviated (Fig. 6), it is observed only at low and high temperatures when $\mathrm{H}_{2} \mathrm{O}$ or $\mathrm{CO}_{2}$ are obviated (Fig. 3, 4 and 5). To explain the behavior of the ignition delay error at medium temperatures in these cases, the chemical effect should be also analyzed.

\subsubsection{Chemical effect}

The chemical effect is caused by the differences in initial concentration of the species, when any of the synthetic EGR are used. A different initial concentration of any species affects the reaction rates and, as a consequence, the relevance of the reactions. All this leads to errors in the ignition delay.

Only two of the three species that can be obviated in the synthetic EGR are chemically reactive $\left(\mathrm{CO}_{2}\right.$ and $\left.\mathrm{H}_{2} \mathrm{O}\right)$. The effects of each of these species on the ignition delay will be explained from a chemical point of view below. A simplified chemical kinetic mechanism was used to find the most relevant 
reactions where the two species mentioned above are involved in. The mechanism used was developed by Patel [41] and it consists of 29 species and 52 reactions. With this reduced mechanism the same trends than with the detailed Curran's mechanism are obtained, both in ignition delay and ignition delay error, for all the simulated types of EGR. Once the most relevant reactions were located, sensitivity analyses of them were performed with Curran's mechanism. A sensitivity analysis of a reaction consists in multiplying the rate constants of this particular reaction by a factor of two (both forward and reverse rates) and then calculating the percent change in ignition delay. Then, those reactions affecting the ignition delay more significantly are found between the most relevant ones. It should be noted that Patel's mechanism was only used to perform the reactions selection, whereas all the calculations were performed with the detailed Curran's mechanism.

The most relevant reaction for the ignition delay where $\mathrm{CO}_{2}$ and $\mathrm{H}_{2} \mathrm{O}$ are involved in is:

$$
\mathrm{H}_{2} \mathrm{O}_{2}+\mathrm{M} \leftrightarrow \mathrm{OH}+\mathrm{OH}+\mathrm{M}
$$

which is a third body reaction where $\mathrm{M}$ can be $\mathrm{H}_{2}, \mathrm{H}_{2} \mathrm{O}, \mathrm{CO}$ or $\mathrm{CO}_{2}$.

$\mathrm{CO}_{2}$ and $\mathrm{H}_{2} \mathrm{O}$ are, therefore, $\mathrm{OH}$ radical generators. This means that while adding $\mathrm{CO}_{2}$ or $\mathrm{H}_{2} \mathrm{O}$ increases the heat capacity of the mixture, which would increase the ignition delay, it also generates free radicals, thus reducing this delay. Regarding the definition of the ignition delay error (Eq. (6)), the impact of the chemical effect on it is very clear: the error increases (becomes more positive) as the mixture is more simplified. Thereby, the chemical and the thermodynamic effects are opposite and the behaviour of the ignition 
delay error will depend on the evolution of the relevance of each one.

Reaction (7) is not very significant at low temperatures due to the lower specific reaction rate (strongly dependent on temperature). It increases its speed at intermediate initial temperatures and it becomes less relevant at high initial temperatures. This trend of the chemical effect, if combined with the termodynamic effect, leads to the existence of a maximum in the ignition delay error at high initial temperatures (e.g. Fig. 5).

The relevance of reaction (7) at intermediate initial temperatures is caused by two factors. First, the specific reaction rate increases due to the temperature increment with respect to the low temperature range. Then, the $\mathrm{H}_{2} \mathrm{O}_{2}$ concentration increases at intermediate temperatures due to the appearance of the NTC regime. The NTC regime appears when the formation of carbonylhydroperoxide radicals and their decomposition into active radicals competes with the formation of olefines from the alkyl and alkylhydroperoxide radicals [4]. This causes the decrease of the reactivity and an increase in the accumulated concentration of $\mathrm{H}_{2} \mathrm{O}_{2}$, promoting the relevance of reaction (7). In fact, the overall reaction rate at the end of the NTC region is controlled by reaction (7) [39].

At high initial temperatures the reaction rate of (7) is found to decrease. This is solely due to the lower concentration of $\mathrm{H}_{2} \mathrm{O}_{2}$, since the concentrations of $\mathrm{CO}_{2}$ and $\mathrm{H}_{2} \mathrm{O}$ before auto-ignition are approximately the same regardless of the initial temperature. Lower concentration of $\mathrm{H}_{2} \mathrm{O}_{2}$ is reached at high initial temperatures for two reasons:

- $\mathrm{H}_{2} \mathrm{O}_{2}$ dissociation. Dissociation of hydrogen peroxide occurs at temperatures of the order of the auto-ignition temperature but with a much 
shorter characteristic time than the ignition delay, thus being an important mechanism of $\mathrm{H}_{2} \mathrm{O}_{2}$ destruction. This dissociation generates two $\mathrm{OH}$ radicals, and consequently the ignition delay is shortened approximately to the same extent as with reaction (7). However, if the hydrogen peroxide dissociates, its reaction with $\mathrm{CO}_{2}$ and $\mathrm{H}_{2} \mathrm{O}$ becomes less relevant and the effect of both species is not so important at high initial temperatures.

- Lower $\mathrm{H}_{2} \mathrm{O}_{2}$ generation. Hydrogen peroxide is created by $\mathrm{HO}_{2}$, mainly by the following reaction:

$$
\mathrm{HO}_{2}+\mathrm{HO}_{2} \leftrightarrow \mathrm{H}_{2} \mathrm{O}_{2}+\mathrm{O}_{2}
$$

At high temperature, the fuel not only suffers dehydrogenation, but it also disappears by decomposition in alkyl radicals $\left(\mathrm{C}_{n} \mathrm{H}_{2 n+1}\right)$ and in hydrocarbon chains of lower molecular weight (thermal cracking) [39]. Thereby, $\mathrm{CH}_{3}$ concentration increases at high initial temperature, promoting the relevance of reaction (9):

$$
\mathrm{CH}_{3}+\mathrm{HO}_{2} \leftrightarrow \mathrm{CH}_{3} \mathrm{O}+\mathrm{OH}
$$

The $\mathrm{CH}_{3}$ preference for $\mathrm{HO}_{2}$ causes a lower formation rate of $\mathrm{H}_{2} \mathrm{O}_{2}$, and its reaction with $\mathrm{CO}_{2}$ and $\mathrm{H}_{2} \mathrm{O}$ becomes less relevant.

For these reasons, obviating $\mathrm{CO}_{2}$ or $\mathrm{H}_{2} \mathrm{O}$ has a very important effect on the medium-temperature range, but a lower one at high temperatures.

The sensitivity analyses show that the following third body reaction has also a high influence on the ignition delay (although much less than reaction 
(7))

$$
H+\mathrm{O}_{2}+\mathrm{M} \leftrightarrow \mathrm{HO}_{2}+\mathrm{M}
$$

where $\mathrm{M}$ can be $\mathrm{H}_{2}, \mathrm{H}_{2} \mathrm{O}, \mathrm{CO}$ or $\mathrm{CO}_{2}$. The hydrogen from the dehydrogenation of the fuel and the molecular oxygen react with the $\mathrm{CO}_{2}$ and the $\mathrm{H}_{2} \mathrm{O}$ to form the active radical $\mathrm{HO}_{2}$. An increment in the concentration of $\mathrm{HO}_{2}$ leads to a faster decomposition of the fuel and, therefore, to a shorter ignition delay. Therefore, if $\mathrm{CO}_{2}$ or $\mathrm{H}_{2} \mathrm{O}$ are obviated, longer ignition delays are obtained. This means that the effect of reaction (10) is the same as the effect of reaction (7). In fact, both lose their relevace at high initial temperatures due to the thermal cracking of the fuel. The higher the thermal cracking, the lower the dehydrogenation of the fuel and the lower the relevance of reaction (10).

The chemical effects of $\mathrm{CO}_{2}$ do not only come from reactions (7) and (10). $\mathrm{CO}_{2}$ destruction can be seen at temperatures where $\mathrm{CO}_{2}$ dissociation is not relevant. This is caused by reactions of the type:

$$
\mathrm{C}_{n} \mathrm{H}_{2 n+1}+\mathrm{CO}_{2} \leftrightarrow \mathrm{C}_{n} \mathrm{H}_{2 n} \mathrm{CO}+\mathrm{OH}
$$

and

$$
\mathrm{CO}_{2}+\mathrm{H} \leftrightarrow \mathrm{OH}+\mathrm{CO}
$$

The sensitivity analysis shows that reaction (11) has no relevant impact on ignition delay. Reaction (12), which is a well known CO oxidation path, affects the ignition delay by competing with the h-abstraction of the fuel $[29,30,35,47]$. Anyway, the relevance of these previous $\mathrm{CO}_{2}$ reactions is negligible compared to reaction (7). Moreover, as it will be seen in Fig. 8, the effects of $\mathrm{CO}_{2}$ are insignificant. It can be clearly seen that water is the key 
species to reproduce the real $E G R$ and its effects are the most important ones.

The different effects on the ignition delay error, caused by obviating both species, are reflected in Fig. 3, where the ignition delay error for the mixture $\mathrm{N}_{2}+\mathrm{O}_{2}$ is shown. At low temperature, the figure shows that the effect of the heat capacity is the dominant one. Consequently, the ignition delay of the simplified mixture is shorter than the delay of the real $E G R$ and, therefore, ignition delay errors are negative. Furthermore, the higher the initial temperature, the higher this thermodynamic effect and the more negative the errors. A change of trend in the ignition delay error can be seen from $850 \mathrm{~K}$. This is caused by the higher relevance of the chemical effects. Now, the reactions where $\mathrm{H}_{2} \mathrm{O}$ and $\mathrm{CO}_{2}$ are involved shorten the auto-ignition. Consequently, the ignition delay of the simplified mixture is longer and the error becomes positive and increases with the initial temperature. However, a new change of trend in the error can be seen from $1000 \mathrm{~K}$. This change is caused by the gradual loss in importance of the chemistry of $\mathrm{H}_{2} \mathrm{O}$ and $\mathrm{CO}_{2}$ (especially of the first), caused by a lower formation of $\mathrm{H}_{2} \mathrm{O}_{2}$ in favor of the reaction of $\mathrm{HO}_{2}$ with $\mathrm{CH}_{3}$, and by a higher dissociation of $\mathrm{H}_{2} \mathrm{O}_{2}$.

These thermodynamic and chemical effects are coherent with those exposed by Fathi et al. [28], Di et al. [31], Dec et al. [26] and Wooldridge et al. [48].

As can be seen in Fig. 3, the maximum ignition delay errors are slightly higher for n-heptane than for isooctane. A more pronounced NTC zone implies a higher accumulated concentration of $\mathrm{H}_{2} \mathrm{O}_{2}$ under the same initial conditions, which promotes the relevance of the chemical effect and leads to 
higher ignition delay errors. Therefore, obviating certain species has a bigger impact on the autoignition process in the case of n-heptane than in the case of isooctane.

\subsection{Effect on the ignition delay error of the equivalence ratio and the oxygen mass fraction}

The ignition delay error becomes more negative when the equivalence ratio is increased, crossing the zero value. The zero crossing is caused by a balance between chemical and thermodynamic effects, for these specific conditions. As it can been seen in Fig. 7, the location of the zero crossing exhibits the following trends:

- The equivalence ratio at which the zero crossing occurs increases if the initial temperature is increased.

- The equivalence ratio at which the zero crossing occurs increases if the pressure is decreased.

- The equivalence ratio at which the zero crossing occurs increases if the oxygen content is decreased.

Fig. 7 also shows that the chemical effect is dominant for lean mixtures. Besides, the richer the equivalence ratio, the more relevant the thermodynamic effect. In the explored range, the chain branching is dependent on radical species formed directly from the fuel, which causes rich mixtures to be more reactive [39]. Thus, the NTC zone becomes less pronounced if the equivalence ratio is increased. A smoother NTC regime implies lower accumulated concentration of $\mathrm{H}_{2} \mathrm{O}_{2}$ and, therefore, a lower relevance of the chemical effects. 

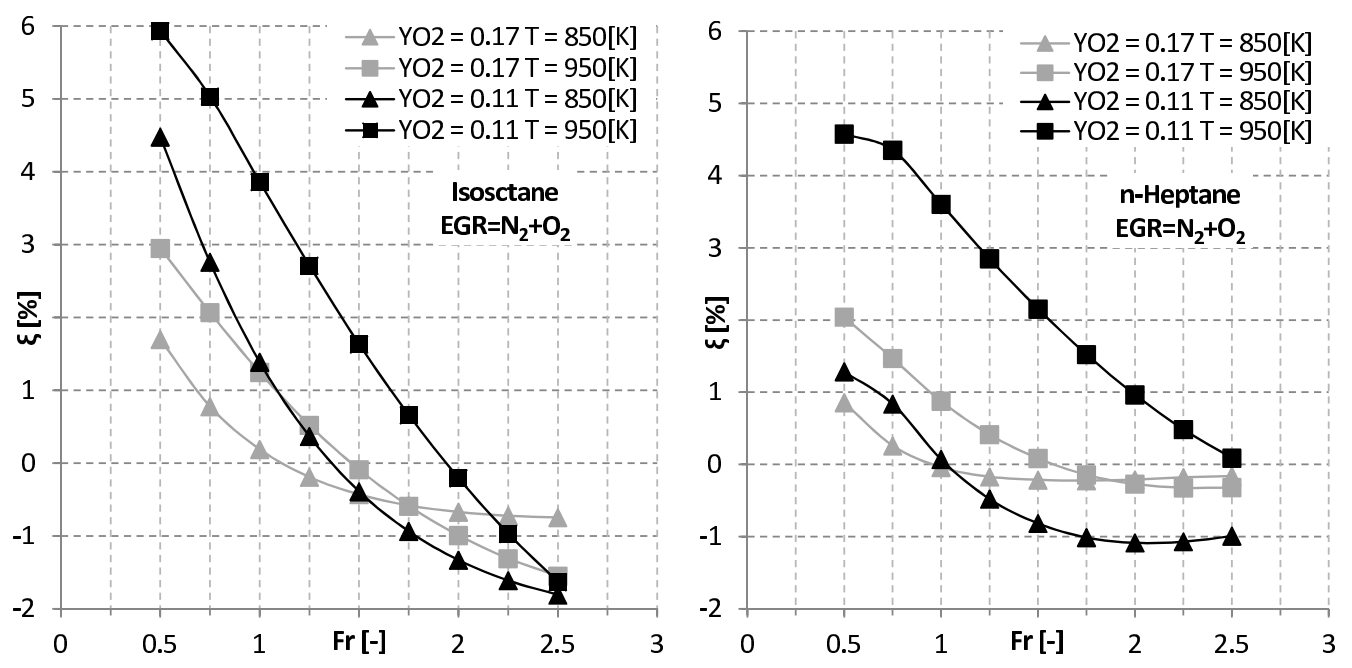

Figure 7: Ignition delay error versus equivalence ratio at $\mathrm{P}=10 \mathrm{MPa}$, different initial temperatures and different oxygen mass fractions. The EGR is formed by $\mathrm{O}_{2}+\mathrm{N}_{2}$. Left.Isooctane. Right.- n-Heptane.

The error in ignition delay increases when the oxygen ratio is decreased, as it can be seen in Fig. 8. Obviously, the more diluted the mixture, the higher the differences between the mole fractions of the different species of one or another type of synthetic EGR. And the more disparate the EGR composition, the greater the error in ignition delay.

Fig. 8 also shows that the prediction accuracy is substantially improved when $\mathrm{CO}_{2}$ and $\mathrm{H}_{2} \mathrm{O}$ are added. In fact, this improvement is higher than the sum of the improvements of adding only one of both species. As far as the authors know, there is not any chemical interaction between $\mathrm{CO}_{2}$ and $\mathrm{H}_{2} \mathrm{O}$ and, due to this chemical independency, the chemical improvement obtained when both species are added is equal than the sum of the improvements. However, due to the exponential dependency of the specific reaction rate with 


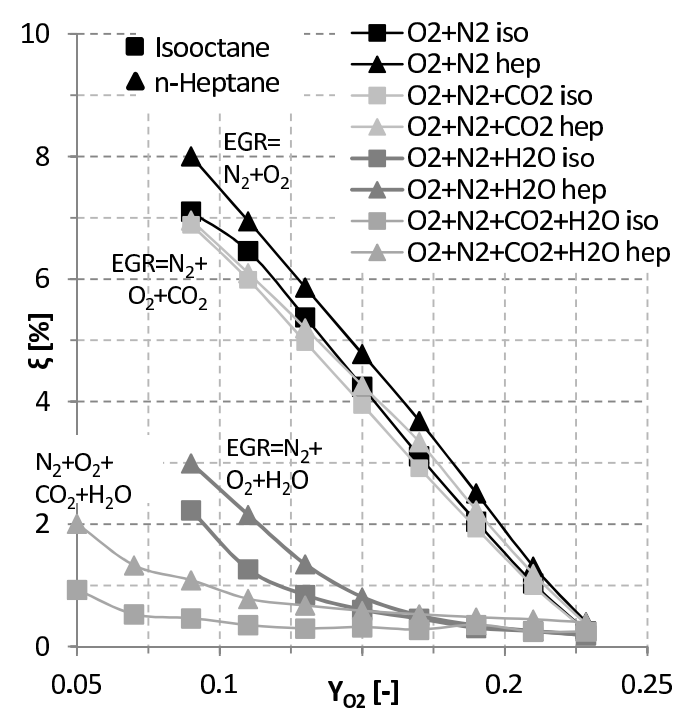

Figure 8: Maximum ignition delay error versus oxygen mass fraction. This is represented for the four simplified mixtures and for both fuels.

temperature, the thermodynamic improvement obtained when both species are taken into account is higher than the sum of the improvements. Thus, a synergistic effect appears between $\mathrm{CO}_{2}$ and $\mathrm{H}_{2} \mathrm{O}$.

Based on these results, the ranges of use of one or another type of synthetic EGR, depending on the desired oxygen mass fraction, can be defined. Establishing that an error in ignition delay higher than $1 \%$ is not admissible, the following global intervals were obtained for the two fuels tested:

- Mixture formed by $\mathrm{N}_{2}+\mathrm{O}_{2}$ : it is valid for oxygen mass fractions greater than or equal to 0.22 .

- Mixture formed by $\mathrm{N}_{2}+\mathrm{O}_{2}+\mathrm{CO}_{2}$ : it is valid for oxygen mass fractions greater than or equal to 0.21 . 
- Mixture formed by $\mathrm{N}_{2}+\mathrm{O}_{2}+\mathrm{H}_{2} \mathrm{O}$ : it is valid for oxygen mass fractions greater than or equal to 0.14 if the fuel used is n-heptane and greater than or equal to 0.12 if the fuel used is isooctane.

- Mixture formed by $\mathrm{N}_{2}+\mathrm{O}_{2}+\mathrm{CO}_{2}+\mathrm{H}_{2} \mathrm{O}$ : in case of using isooctane, it is valid for all the oxygen mass fractions simulated. In case of using n-heptane, it is valid for oxygen mass fractions greater than or equal to 0.09 .

The criterion adopted here to give validity to each of the studied simplifications is completely arbitrary. This is a relatively strict criterion, but serves as a reference to select one synthetic mixture or another, ensuring their reality is accurately reproduced. Setting any other error limit, the new ranges of use can be obtained from Fig. 8.

It is also noted that it is not necessary to add up argon to oxygen mass fractions less than or equal to 0.09 for the case of n-heptane, and in any case when using isooctane, since the limit fraction of auto-ignition $\left(\mathrm{Y}_{\mathrm{O}_{2}}=0.05\right)$ has been reached without exceeding $1 \%$ error in ignition delay (which is the criterion used in this investigation for discarding a simplified mixture).

\section{Conclusions}

In this work a method to create synthetic mixtures simulating the exhaust gas recirculation of an engine has been designed. These mixtures can later be used in experimental facilities to study the auto-ignition phenomenon in both standard and low temperature conditions.

The method presented is based on using five different species: $\mathrm{N}_{2}, \mathrm{O}_{2}$, 
$\mathrm{CO}_{2}, \mathrm{H}_{2} \mathrm{O}$ and Ar. However, it is possible to work with the following simplified mixtures, with errors in the ignition delay of less than $1 \%$ :

- Mixture formed by $\mathrm{N}_{2}+\mathrm{O}_{2}: \mathrm{Y}_{O_{2}} \geq 0.22$.

- Mixture formed by $\mathrm{N}_{2}+\mathrm{O}_{2}+\mathrm{CO}_{2}: 0.22 \geq \mathrm{Y}_{\mathrm{O}_{2}} \geq 0.21$.

- Mixture formed by $\mathrm{N}_{2}+\mathrm{O}_{2}+\mathrm{H}_{2} \mathrm{O}: 0.21 \geq \mathrm{Y}_{O_{2}} \geq 0.14$.

- Mixture formed by $\mathrm{N}_{2}+\mathrm{O}_{2}+\mathrm{CO}_{2}+\mathrm{H}_{2} \mathrm{O}: 0.14 \geq \mathrm{Y}_{O_{2}} \geq 0.09$.

Ignition delay changes when any species is obviated due to a combination of two opposite effects, a thermodynamic and a chemical effect. The thermodynamic effect leads to shorter ignition delays when a species is obviated. Besides, this effect predominates at low and at high initial temperatures and for rich mixtures. The chemical effect leads to longer ignition delays when a specie is obviated. This effect predominates at medium initial temperatures and for lean mixtures.

To conclude, in order to create an oxygen-lean mixture to study the autoignition phenomenon of isooctane and/or n-heptane, under real conditions, the following species have to be taken in account: $\mathrm{N}_{2}, \mathrm{O}_{2}, \mathrm{CO}_{2}$ and $\mathrm{H}_{2} \mathrm{O}$. Argon can be obviated for oxygen mass fractions higher than 0.09 with ignition delay errors lower than $1 \%$. Furthermore, it is not necessary to add $\mathrm{CO}_{2}$ when the proportion of oxygen reaches medium-high values $(\geq 0.14)$. This is possible because $\mathrm{H}_{2} \mathrm{O}$ is, by far, the species with the major influence on the ignition delay for the proportions used in this study. 


\section{Acknowledgements}

The authors would like to thank different members of the CMT-Motores Térmicos team of the Universitat Politècnica de València for their contribution to this work. The authors would also like to thank the Spanish Ministry of Education for financing the PhD. Studies of Darío López-Pintor (grant FPU13/02329). This work was partly founded by the Generalitat Valenciana, project PROMETEOII/2014/043.

\section{Notation}

$a_{s t} \quad$ Oxygen-fuel ratio under stoichiometric conditions

CI Compression Ignition

EGR Exhaust Gas Recirculation

F $\quad$ Fuel to oxygen ratio

$\mathrm{Fr} \quad$ Working equivalence ratio

HCCI Homogeneous Charge Compression Ignition

$H R R \quad$ Heat Release Rate

LTC Low Temperature Combustion

$m \quad$ Number of hydrogens in a molecule of fuel

$M_{i} \quad$ Mass of the $i$ species

$M W_{i} \quad$ Molecular weight of the $i$ species

$n \quad$ Number of carbons in a molecule of fuel

NTC Negative Temperature Coefficient

$p \quad$ Number of oxygens in a molecule of fuel 
$P \quad$ Pressure

PCCI Premixed Charge Compression Ignition

PRF Primary Reference Fuels

PSR Perfectly Stirred Reactor

realEGR Referred to the EGR formed by $\mathrm{O}_{2}, \mathrm{~N}_{2}, \mathrm{CO}_{2}, \mathrm{H}_{2} \mathrm{O}$ and $\mathrm{Ar}$

SI Spark Ignition

TDC Top Dead Center

T Temperature

UHC Unburned hydrocarbons

$X_{i} \quad$ Molar fraction of the $i$ species

$Y_{i} \quad$ Mass fraction of the $i$ species

$\xi_{i} \quad$ Ignition delay error of the EGR $i$

$\Delta T \quad$ Referred to the criterion whereby the auto-ignition of the mixture is considered to be produced when the temperature in the reactor increases $400 K$ over the initial temperature

$\nabla T \quad$ Referred to the criterion whereby the auto-ignition of the mixture is considered to be produced when the maximum temperature gradient occurs

$\phi \quad$ Auxiliary equivalence ratio

$\tau_{i} \quad$ Ignition delay of the EGR $i$ 


\section{Subscripts}

atm Referred to atmospheric conditions

$f \quad$ Referred to the fuel

mixture Referred to the synthetic EGR + fuel mixture

products Referred to the products of a complete combustion reaction of a certain fuel with dry air

realEGR Referred to the EGR formed by $\mathrm{O}_{2}, \mathrm{~N}_{2}, \mathrm{CO}_{2}, \mathrm{H}_{2} \mathrm{O}$ and $\mathrm{Ar}$ st $\quad$ Referred to stoichiometric conditions

\section{References}

[1] J.B. Heywood. Internal combustion engine fundamentals. Mc Graw Hill, 1988.

[2] T. Li, D. Wu, and M. Xu. Thermodynamic analysis of EGR effects onthe firstand second law efficiencies ofaboosted spark-ignited direct-injection gasoline engine. Energy Conversion and Management, 70:130-138, 2013.

[3] U. Asad, J. Tjong, and M. Zheng. Exhaust gas recirculation - Zero dimensional modelling and characterization for transient diesel combustion control. Energy Conversion and Management, 86:309-324, 2014.

[4] X.C. Lü, W. Chen, and Z. Huang. A fundamental study on the control of the HCCI combustion and emissions by fuel design concept combined 
with controllable EGR. Part 1. The basic characteristics of HCCI combustion. Fuel, 84:1083-1974, 2005.

[5] Z. Zheng, L. Yue, H. Liu, Y. Zhu, X. Zhong, and M-Yao. Effect of two-stage injection on combustion and emissions under high EGR rate on a diesel engine by fueling blends of diesel/gasoline, diesel/n-butanol, diesel/gasoline/n-butanol and pure diesel. Energy Conversion and Management, 90:1-11, 2015.

[6] X.C. Lü, W. Chen, and Z. Huang. A fundamental study on the control of the HCCI combustion and emissions by fuel design concept combined with controllable EGR. Part 2. Effect of operating conditions and EGR on HCCI combustion. Fuel, 84:1092-1984, 2005.

[7] S.S. Nathan, J.M. Mallikarjuna, and A. Ramesh. An experimental study of the biogas-diesel HCCI mode of engine operation. Energy Conversion and Management, 51:1347-1353, 2010.

[8] K. Bahlouli, U. Atikol, R.K. Saray, and V. Mohammadi. A reduced mechanism for predicting the ignition timing of a fuel blend of naturalgas and n-heptane in HCCI engine. Energy Conversion and Management, 79:85-96, 2014.

[9] J.C.G. Andrae. Development of a detailed kinetic model for gasoline surrogate fuels. Fuel, 87:2013-2022, 2008.

[10] X.C. Lü, Y. Hou, L. Zu, and Z. Huang. Experimental study on the autoignition and combustion characteristics in the homogeneous charge com- 
pression ignition (HCCI) combustion operation with ethanol/n-heptane blend fuels by port injection. Fuel, 85:2622-2631, 2006.

[11] P. Dagaut, A. Ristori, A. El Bakali, and M. Cathonnet. Experimental and kinetic modeling study of the oxidation of n-propylbenzene. Fuel, $81: 173-184,2002$.

[12] H. Nakamura, D. Darcy, M. Mehl, C.J. Tobin, W.K. Metcalfe, W.J. Pitz, C.K. Westbrook, and H.J. Curran. An experimental and modeling study of shock tube and rapid compression machine ignition of n-butylbenzene/air mixtures. Combustion and Flame, 161:49-64, 2014.

[13] M. Bardi, R. Payri, L.M. Malbec, G. Bruneaux, L.M. Pickett, J. Manin, T. Bazyn, and C. Genzale. Engine Combustion Network: comparison of spray development, vaporization, and combustion in different combustion vessels. Atomization and Sprays, 22(10):807-842, 2012.

[14] M. Sjöberg and J.E. Dec. An investigation into lowest acceptable combustion temperatures for hydrocarbon fuel in HCCI engines. Proceedings of the Combustion Institute, 30:2719-2726, 2005.

[15] M. Sjöberg and J.E. Dec. Isolating the effects of fuel chemistry on combustion phasing in an HCCI engine and the potential of fuel stratification for ignition control. SAE Paper no. 2004-01-0557, 2004.

[16] R. Minetti, M. Carlier, M. Ribacour, E. Therssen, and L.R. Sochet. Comparison of oxidation and autoignition of the two Primary Reference Fuels by rapid compression. Twenty-Sixth Symposium (International) on Combustion/The Combustion Institute, pages 747-753, 1996. 
[17] B.M. Gauthier, D.F. Davidson, and R.K. Hanson. Shock tube determination of ignition delay times in full-blend and surrogate fuel mixtures. Combustion and Flame, 139:300-311, 2004.

[18] N. Ladommatos, S. Abdelhalim, H. Zhao, and Z. Hu. The dilution, chemical, and thermal effects of Exhaust Gas Recirculation on diesel engine emissions - Part 1: Effect of reducing inlet charge oxygen. SAE Paper no. 961165, 1996.

[19] N. Ladommatos, S. Abdelhalim, H. Zhao, and Z. Hu. The dilution, chemical, and thermal effects of Exhaust Gas Recirculation on diesel engine emissions - Part 2: Effects of carbon dioxide. SAE Paper no. 961167, 1996.

[20] N. Ladommatos, S. Abdelhalim, H. Zhao, and Z. Hu. The dilution, chemical, and thermal effects of Exhaust Gas Recirculation on diesel engine emissions - Part 3: Effects of water vapour. SAE Paper no. 971659, 1997.

[21] N. Ladommatos, S. Abdelhalim, H. Zhao, and Z. Hu. The dilution, chemical, and thermal effects of Exhaust Gas Recirculation on diesel engine emissions - Part 4: Effects of carbon dioxide and water vapour. SAE Paper no. 971660, 1997.

[22] H. Zhao, Z. Peng, J. Williams, and N. Ladommatos. Understanding the effects of recycled burnt gases on the Controlled Autoignition (CAI) combustion in four-stroke gasoline engines. SAE Paper no. 2001-013607, 2001. 
[23] J.E. Dec. A computational study of the effects of low fuel loading and EGR on heat release rates and combustion limits in HCCI engines. $S A E$ Paper no. 2002-01-1309, 2002.

[24] R. Chen and N. Milovanovic. A computational study into the effect of exhaust gas recycling on homogeneous charge compression ignition combustion in internal combustion engines fuelled with methane. International Journal of Thermal Sciences, 41:805-813, 2002.

[25] M. Sjöberg, J. Dec, and W. Hwang. Thermodynamic and chemical effects of EGR and its constituents on HCCI autoignition. SAE Paper no. 2007-01-020\%, 2007.

[26] M. Sjöberg and J.E. Dec. Effects of EGR and its constituents on HCCI autoignition of ethanol. Proceedings of the Combustion Institute, 33:3031-3038, 2011.

[27] A. Babajimopoulos, G.A. Lavoie, and D.N. Assanis. Modeling HCCI combustion with high levels of residual gas fraction. A comparison of two VVA strategies. SAE Paper no. 2003-01-3220, 2003.

[28] M. Fathi, R.K. Saray, and M.D. Checkel. The influence of Exhaust Gas Recirculation (EGR) on combustion and emissions of n-heptane/natural gas fueled Homogeneous Charge Compression Ignition (HCCI) engines. Applied Energy, 88:4719-4724, 2011.

[29] T. Le Cong and P. Dagaut. Experimental and detailed kinetic modeling of the oxidation of natural gas, natural gas/syngas mixtures, and effect of burnt gas. Proceedings of the European Combustion Meeting, 2007. 
[30] J. Herzler and C. Naumann. Shock tube study of the influence of NOx on the ignition delay times of natural gas at high pressure. Combustion Science and Technology, 184:1635-1650, 2012.

[31] H. Di, X. He, P. Zhang, Z. Wang, M.S. Wooldridge, C.K. Law, C. Wang, S. Shuai, and J. Wang. Effects of buffer gas composition on low temperature ignition of iso-octane and n-heptane. Combustion and Flame, 161:2531-2538, 2014.

[32] B.W. Weber, K. Kumar, Y. Zhang, and C.J. Sung. Autoignition of nbutanol at elevated pressure and low-to-intermediate temperature. Combustion and Flame, 158:809-819, 2011.

[33] G. Mittal, M. Chaos, C.J. Sung, and F.L. Dryer. Dimethyl ether autoignition in a rapid compression machine: Experiments and chemical kinetic modeling. Fuel Processing Technology, 89:1244-1254, 2008.

[34] G. Kukkadapu, K. Kumar, C.J. Sung, M. Mehl, and W.J. Pitz. Autoignition of gasoline and its surrogates in a rapid compression machine. Proceedings of the Combustion Institute, 34:345-352, 2013.

[35] J.M. Anderlohr, A. Piperel, A. Pires da Cruz, R. Bounaceur, F. BattinLeclerc, P. Dagaut, and X. Montagne. Influence of EGR compounds on the oxidation of an HCCI-diesel surrogate. Proceedings of the Combustion Institute, 32:2851-2859, 2009.

[36] H. Machrafi. Experimental validation of a kinetic multi-component mechanism in a wide HCCI engine operating range for mixtures of n- 
heptane, iso-octane and toluene: Influence of EGR parameters. Energy Conversion and Management, 49:2956-2965, 2008.

[37] A. Frassoldati, T. Faravelli, and E. Ranzi. Kinetic modeling of the interactions between NO and hydrocarbons at high temperature. Combustion and Flame, 135:97-112, 2003.

[38] H.J. Curran, P. Gaffuri, W. J. Pitz, and C. K. Westbrook. A comprehensive modeling study of iso-octane oxidation. Combustion and Flame, 129:253-280, 2002.

[39] H.J. Curran, P. Gaffuri, Pitz W.J, and C.K. Westbrook. A comprehensive modeling study of n-heptane oxidation. Combustion and Flame, 114:149-177, 1998.

[40] H.J. Curran, W.J. Pitz, C.K. Westbrook, C.V. Callahan, and F.L. Dryer. Oxidation of automotive primary reference fuels at elevated pressures. Proceedings of the Combustion Institute, 27:379-387, 1998.

[41] A. Patel, S. C. Kong, and R. D. Reitz. Development and validation of a reduced reaction mechanism for HCCI engine simulations. SAE Technical Paper Series, 2004-01-0558, 2004.

[42] F. Payri, X. Margot, S. Patouna, F. Ravet, and M. Funk. Use of a single-zone thermodynamic model with detailed chemestry to study a natural gas fueled Homogeneous Charge Compression Ignition engine. Energy Conversion and Management, 53:298-304, 2012.

[43] P. Redón. Modelling of the nitogen oxides formation process applicable 
to several diesel combustion modes. PhD thesis, Universitat Politècnica de València, Valencia (Spain), 2013.

[44] S. Jerzembeck, N. Petersa, P. Pepiot-Desjardins, and H. Pitsch. Laminar burning velocities at high pressure for primary reference fuels and gasoline: Experimental and numerical investigation. Combustion and flame, 156:292-301, 2009.

[45] E. Mastorakos. Ignition of turbulent non-premixed flames. Progress in Energy and Combustion Science, 35:57-97, 2009.

[46] M.E. Baumgardner, S.M. Sarathy, and A.J. Marchese. Autoignition characterization of primary reference fuels and n-heptane/n-butanol mixtures in a constant volume combustion device and Homogeneous Charge Compression Ignition engine. Energy and Fuels, 27(12):77787789, 2013.

[47] P. Glarborg and L.L.B. Bentzen. Chemical effects of high CO2 concentration in oxy-fuel combustion of methane. Energy and Fuels, 22:291$296,2008$.

[48] S.W. Wagnon and M.S. Wooldrdge. Effects of buffer gas composition on autoignition. Combustion and Flame, 161:898-907, 2014. 